\title{
Stellar nonlinear dynamos: observations and modelling
}

\author{
Ilkka Tuominen ${ }^{1}$, Maarit J. Korpi ${ }^{1}$, Petri J. Käpylä ${ }^{1}$, \\ Marjaana Lindborg ${ }^{1}$, and Ilya Ilyin ${ }^{2}$ \\ ${ }^{1}$ Observatory, University of Helsinki, PO BOX 14, FI-00014 University of Helsinki, Finland \\ ${ }^{2}$ Astrophysical Institute Potsdam, An der Sternwarte 16, D-14482 Potsdam, Germany
}

\begin{abstract}
Recent numerical modelling of mean-field stellar dynamos with induction and Reynolds' equations show that, with increasing rotation, field symmetry changes from an axisymmetric solar type to a nonaxymmetric one, where the so-called active longitudes in the same stellar hemisphere are predicted to be of opposite polarities. It was originally named Active star Hale rule in Tuominen et al. (2002), being an analogue to the famous bipolar sunspot polarity rule but different in scale and being a global phenomenon. In addition to long timeseries of temperature mapping and photometry, during the last few years we have been able to measure accurately polarization spectra of an active late-type star II Peg with the upgraded spectropolarimetric option of the high-resolution spectrograph SOFIN at the Nordic Optical Telescope, La Palma. The magnetic inversions (Carroll et al., Kochukhov et al.) can be compared to the dynamo models and the preliminary results show some resemblance to the dynamo solutions.
\end{abstract}

Keywords. Stars:late-type - stars: spots - MHD - methods: numerical

\section{Introduction}

Recently Käpylä et al. (2006) performed local convection calculations to determine the $\alpha$-tensor resulting from turbulent convection. They utilized the so-called imposed field method, where weak uniform magnetic fields into certain direction are imposed on the flow, and the corresponding $\alpha$-tensor components solved from the series expansion of the turbulent electromotive force. Calculations at varying latitude and angular velocity were performed. At the highest angular velocity, corresponding to the Coriolis number of $\approx 10$, the $\alpha_{\phi \phi}$ component is observed no longer to peak at the pole as conventionally assumed, but to be concentrated near the equatorial region, following approximately a $\sin ^{2} \theta \cos \theta$-dependence. Here we test corresponding profiles in a mean-field dynamo calculation.

\section{Nonaxisymmetric mean-field model}

We solve for the mean-field induction and Reynolds'equations in $2 \frac{1}{2}$ dimensions, meaning that the azimuthal direction is treated in Fourier space (see e.g. Moss et al. 1995). The equations are solved in an incompressible fluid shell $R_{0}<r<1, \pi / 2<\theta<-\pi / 2$, for the lowest four Fourier modes $m=0, \ldots, 3$. Here we take $R_{0}=0.5$. We adopt a simple model for the Lambda-effect, namely $\Lambda_{V}=\nu_{T} V^{0}$ while other components vanish; here we use $V^{0}=1$. Different types of latitudinal $\alpha$-profiles are used, motivated by the local convection simulations. Similar study has been conducted for axisymmetric $\alpha^{2}$-dynamo model by Rüdiger et al. (2003); our model differs in that we also consider nonaxisymmetric modes, include some (but only very little) differential rotation, and consider only isotropic $\alpha$-effect. 

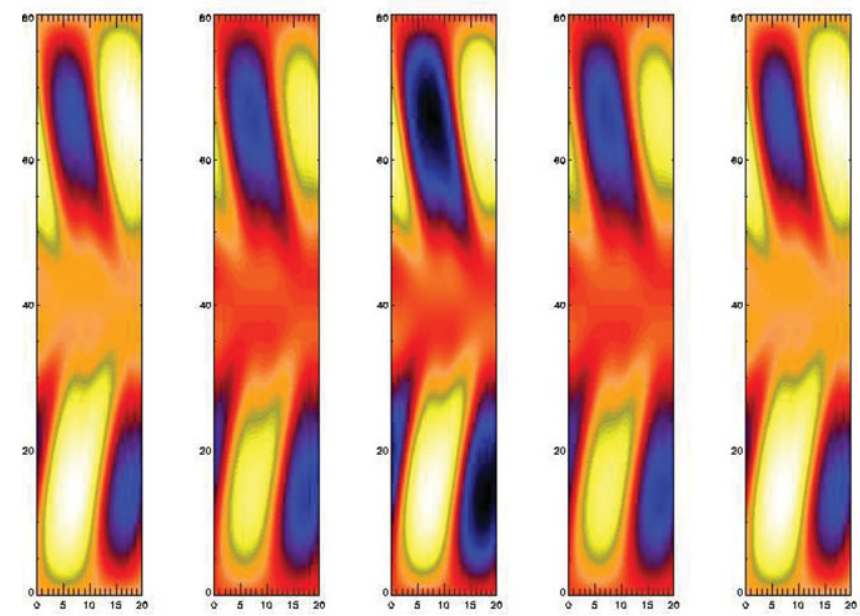

Figure 1. Radial field at the surface for the run $C_{\alpha 1}=100, T a=10000$ at five different timepoints covering the whole oscillation cycle. The small and rapid oscillation is related to the energy levels of the spots, while the polarity of the spots remains unchanged. The overall appearance of the instantaneous dynamo solution shows some resemblance to the observed magnetic field configuration (Carroll et al. 2009, Kochukhov et al. 2009).

\section{Results}

As reported in our earlier papers (Tuominen et al. 1999, 2002), the simplest $\alpha$-profile leads to $A 1$-type solutions at rapid rotation i.e. high Taylor numbers $(T a)$. Due to $V^{0} \neq 0$, some radial differential rotation is generated; the absolute differential rotation, however, is small, $|\Delta \Omega|<100$ for the largest values of $T a$ investigated. The dominating Fourier mode $m=1$ is always drifting in azimuth with respect to the rotational frame so that for $T a<1000$ the mode is lagging and for $T a>1000$ going ahead the rotational frame. The higher $T a$, the closer the poles the spots appear. There is a small oscillation at high $T a$ related to the relative energy levels of the spots; the spots with negative radial field get simultaneously weaker while the positive ones gets stronger and vice versa; this behaviour is depicted in Fig.1. The timescale of this variation is very short compared to the diffusion time (typically $<0.1$ diffusion times).

Local convection calculations suggest that the $\alpha$-effect would be concentrated to the equatorial regions with rapid rotation. Implementing profiles of this type to the nonaxisymmetric mean-field dynamo model including dynamics and small amounts of differential rotation showed that axisymmetric modes can coexist with the nonaxisymmetric modes even in the rapid rotation regime, mixed modes and parities are typical, and solutions can be oscillatory.

\section{References}

Carroll, T., Kopf, M., Strassmeier, K., Ilyin, I., \& Tuominen, I., 2009, this proceedings Kochukhov, O., Piskunov, N., Ilyin, I., \& Tuominen, I., 2009, this proceedings Käpylä, P.J., Korpi, M.J., Ossendrijver, M., \& Stix, M. 2006, A\&A 455, 401 Moss, D., Barker, D. M., Brandenburg, A., \& Tuominen I. 1995, A\& A 294, 155 Rüdiger, G., Elstner, D., \& Ossendrijver, M. 2003, A\&ऽA 406, 15

Tuominen, I., Bergyugina, S. V., Korpi, M. J., \& Rönty, T. 1999, in Stellar dynamos: Nonlinearity and Chaotic Flows, Nunez \& Ferriz-Mas (eds.), ASP Conference Series 178

Tuominen, I., Berdyugina, S. V., \& Korpi, M. J. 2002, AN 323, 367 